\title{
GERENCIAMENTO DE FARMÁCIA HOSPITALAR: ENFOQUE LOGÍSTICO NA CADEIA DE SUPRIMENTOS
}

\section{ENSAIO TEÓRICO}

OLIVEIRA, Rayanne Alves De ${ }^{1}$

LIMA, Vinícius Mende ${ }^{2}$

FRANÇA, Lucielma Cavalcante De Jesus ${ }^{3}$

SILVA, Lucrécia Pereira ${ }^{4}$

OLIVEIRA, Rayanne Alves De. Et al. Gerenciamento de farmácia hospitalar: Enfoque logístico na cadeia de suprimentos. Revista Científica Multidisciplinar Núcleo do Conhecimento. Ano 05, Ed. 09, Vol. 03, pp. 87-98. Setembro de 2020. ISSN: 2448-0959, Link de acesso: https://www.nucleodoconhecimento.com.br/saude/farmacia-hospitalar

\section{RESUMO}

O hospital é uma unidade crítica no processo de gestão de forma geral. Essa criticidade se torna mais acentuada quando se refere à logística de suprimentos. $\mathrm{Na}$ unidade hospitalar, convém destacar a farmácia hospitalar como um núcleo indispensável para um tratamento eficaz e seguro ao paciente. Objetivo: identificar e

1 Pós-graduanda em controle de infecção hospitalar pela FUTURA. Especialista em Saúde Mental pelo INESPO, Bacharel em Enfermagem pela Universidade Federal do Maranhão- UFMA.

${ }^{2}$ Graduado em Tecnologia de Logística pela Universidade Federal do Tocantins-UFT. ${ }^{3}$ Especialista em Obstetrícia e Neonatologia pelo CEUMA. Bacharel em enfermagem pela Faculdade de Educação São Francisco.

${ }^{4}$ Especialista em Enfermagem do Trabalho, UTI e Urgência e Emergência pelo INESPO. Bacharel em Enfermagem pela Universidade Federal do Maranhão -UFMA. 
relatar as principais dificuldades e ineficiências no processo da gestão de suprimentos em farmácias hospitalares. Metodologia: Revisão literária de artigos indexados nas bases de dados Lilacs, SPELL, Scielo, Medline e Portal de Periódicos CAPES, no período de 2005 a 2019, sobre gestão de suprimentos em farmácias hospitalares. Resultados: foram selecionados 25 artigos, que abordaram a temática, os quais foram estudados criticamente, visando compreender o processo da cadeia de suprimentos nas farmácias hospitalares. Conclusão: a principal dificuldade encontrada foi o controle eficaz dos estoques. Em consequência disso, em dado momento, a falta de alguns materiais e medicamentos, trazendo, portanto, danos ou prejuízos aos pacientes.

Palavras-chave: Serviço de farmácia hospitalar, central hospitalar de suprimentos, administração de materiais no hospital.

\section{INTRODUÇÃO}

As unidades hospitalares detêm variável quantidade de áreas do conhecimento necessárias na manutenção das suas atividades, que devem ser conduzidas de forma apropriada para que os serviços de saúde sejam executados com a maior eficácia possível. Com isso percebe-se o motivo pelo qual é uma área de tamanha complexidade (PRESTES, 2019).

A gestão de hospitais, independente de qual região ele esteja, exige do profissional que o administra diversos conhecimentos, dentre eles conhecimento sobre regulação, financiamento, tecnologias em saúde, gerenciamento de recursos físicos, humanos e financeiros (FARIAS; ARAÚJO, 2016).

Segundo Sousa (2011), mesmo diante da evolução gradual na gerência desse tipo de organização, o aprimoramento da eficiência da logística de abastecimento dos estabelecimentos hospitalares e o controle efetivo dos custos, continua a ser um enorme desafio a ser superado na saúde, tendo em vista a peculiaridade dos serviços prestados e a diversidade de materiais usados em sua realização. 
Tendo como ponto central a minimização do custo da operação para determinado nível de serviço, a logística é por demais importante no processo da viabilização do fluxo de suprimento de materiais. Ela almeja alcançar o nível esperado do serviço ao cliente com o menor custo final (PEREIRA, 2006).

De acordo com a Resolução no 492 do Conselho Federal de Farmácia (2008), a farmácia hospitalar é definida como "unidade clínica, administrativa e econômica, dirigida por farmacêutico, ligada hierarquicamente à direção do hospital ou serviço de saúde e integrada funcionalmente com as demais unidades administrativas e de assistência ao paciente".

A farmácia hospitalar faz parte da estrutura do hospital e tem por meta principal assegurar a excelência da assistência que chega ao paciente, sendo responsável pela promoção do uso seguro e racional medicamentos e insumos hospitalares, prescritos pelo profissional médico, além de atender as demandas e necessidades de medicamentos dos pacientes internados no hospital. Para tanto, ela precisa manter sob sua guarda os estoques desses produtos (CAVALLINI; BISSON, 2010).

Neste contexto, percebe-se que os fármacos possuem grande repercussão na gestão logística, sendo estes recursos de significativa importância no processo de atendimento e cuidado ao paciente, além de serem considerados fatores determinantes nas despesas de unidades de saúde (NETO; FILHO, 1998).

A gestão da cadeia de suprimentos abrange algumas etapas, dentre elas estão o planejamento, a organização, o apontamento e o domínio de todas as etapas existentes no processo de otimização de valor de toda essa cadeia. Deste modo, compreende os fluxos desde a fase em que se adquire os insumos, suas modificações e fases de distribuição do produto até o alcance do mesmo pelo usuário final. (ACCIOLY; AYRES; SUCUPIRA, 2008).

A logística hospitalar tem importante notoriedade pelo motivo de que um erro na distribuição dos materiais e medicamentos, uma função logística que é primordial, pode levar consequentemente a um irremediável desastre, atingindo não somente o 
hospital, mas também aos pacientes (BARBUSCIA, 2006 apud OLIVEIRA; MUSSETTI, 2014).

Este estudo objetivou, portanto, identificar e relatar as principais dificuldades e ineficiências no processo da gestão de suprimentos em farmácias hospitalares. Justifica-se, por tal cenário exposto, a necessidade de estudos que como este se destinem a investigar como ocorre o fluxo logístico em farmácias hospitalares, devido à complexidade desse tipo estoque e o dano que a ineficiência no processo de gestão do mesmo pode causar no paciente.

\section{METODOLOGIA}

Trata-se de um estudo exploratório, descritivo, feito por meio de levantamento bibliográfico de artigos indexados nas bases de dados Lilacs, SPELL, Scielo; Medline, e Portal de Periódicos CAPES, no período de 2005 a 2019, sobre gestão de suprimentos em farmácias hospitalares, para busca utilizou-se os seguintes descritores: serviço de farmácia hospitalar; central hospitalar de suprimentos; administração de materiais no hospital.

Na pesquisa foram selecionados artigos, com essa abordagem temática, os artigos selecionados passaram por uma análise crítica, com o objetivo de identificar e relatar as principais dificuldades e ineficiências no processo da gestão de suprimentos em farmácias hospitalares.

Nesta revisão adotou-se como critério de inclusão estudos que comtemplaram a temática buscada, realizados nos últimos 14 anos, e como critério de exclusão estudos com mais de 14 anos de publicação ou que não se relacionassem com os descritores pesquisados.

Dentro dos critérios de inclusão e exclusão na seleção, na busca final classificou-se para análise neste estudo um total de vinte e cinco (25) artigos que estavam relacionados diretamente ao tema e objetivos do estudo, contemplando os critérios 
esperados. Tais artigos foram lidos e relidos tornando possível a sua síntese, interpretação e análise crítica.

\section{REVISÃO LITERÁRIA}

A administração de materiais é formada por etapas logísticas, dentre as quais estão a escolha dos materiais e fornecedores, a aquisição, o recebimento, o controle e organização dos materiais em estoque, o armazenamento, a dispensação e a assistência ao público destinado (BARBIERI; MACHLINE, 2017).

Essa administração se aplica também na área de saúde, mais precisamente ao setor de farmácia hospitalar, ao qual é incumbido fazer toda a gestão dos seus materiais e medicamentos, que trazem consigo uma enorme variação de itens e grandes dimensões no quesito de quantidade. Conforme nos relata Barbuscia (2006), quando diz que a "Logística é uma função da administração de materiais responsável por disponibilizar materiais e medicamentos no tempo requerido para atender os pacientes"

Com isto evidencia-se que, a logística direciona todas as fases pelas quais passam os materiais e informações na cadeia de suprimentos, desde os fornecedores dos materiais até a entrega aos pacientes, atuando em cada fase do processo, para assegurar que não haja falhas nesse processo ou que elas sejam as mínimas possíveis.

A logística de distribuição nos hospitais tem como dever garantir a entrega de medicamentos e materiais no tempo certo e no setor solicitado. Entretanto, nessas organizações frequentemente o fluxo de distribuição não é bem preciso e nem sempre é possível quantificar de forma exata todos os materiais que serão necessários em algum procedimento médico. Essa deficiência na previsão pode acarretar dificuldades de planejamento e controle (PEREIRA, 2006).

A farmácia hospitalar caracteriza-se por ser um setor com habilidades clínicas e assistenciais, que possui capacidade de gerência e administração, atuando dessa 
forma como um dos principais setores do hospital. Tem em sua responsabilidade o abastecimento seguro e racional dos medicamentos e alguns artigos médicohospitalares, podendo vincular-se a diretoria clínica ou administrativa do estabelecimento hospitalar (SILVA et al., 2013).

Ela possui diversas funções, abrangendo desde as atividades comuns do ciclo de assistência farmacêutica como seleção, programação, aquisição, armazenamento, distribuição e dispensação de medicamentos, como também o gerenciamento de recursos humanos, financeiros e materiais (SILVA et al., 2013).

Os estoques são elementos essenciais para o atendimento as demandas previsíveis, são eles que abastecem todo o fluxo produtivo, permitindo dessa forma, racionalizações nos processos de compra, assegurando uniformidade em processos produtivos e tornam possível as instituições a prática de economia. Por essa razão, os estoques podem ser vistos como elementos intrinsecamente associados à competitividade das organizações (ACCIOLY; AYRES; SUCUPIRA, 2008).

Conforme Cavallini e Bisson (2010), os estoques nas farmácias hospitalares são caracterizados por ciclos de demandas e ressuprimentos, com variações frequentes e altos graus de instabilidades, fatores críticos mediante a necessidade de se armazenar medicamentos em disponibilidade na mesma proporção em que estes são utilizados. Esses medicamentos em grandes estoques representam altos custos, e nas instituições hospitalares os medicamentos e materiais podem significar até $75 \%$ dos gastos financeiros.

A gestão da cadeia de suprimento constitui um processo indispensável para alcançar resultados satisfatórios entre empresas e clientes, de forma que ela torna possível o controle de todos os processos, redução de custos maiores e disponibilidade do produto quando necessário.

Ela constitui a atividade de planejar, implementar e controlar o fluxo e armazenamento econômico de matérias-primas, materiais semiacabados e produtos acabados, bem como as situações a eles relativas, desde o ponto-de-origem até o ponto-de-consumo, 
tendo por finalidade satisfazer os interesses dos clientes (BARBIERI; MACHLINE, 2017).

Com isto, para um adequado dimensionamento de estoque, que atenda bem o usuário final é preciso avaliar todo fluxo e trâmites que os medicamentos e materiais passam antes de estarem disponíveis nas farmácias hospitalares, conhecendo todo esse fluxo, desde produção, disponibilidade em grandes empresas e representantes, solicitação, pedido de compra, transporte e entrega, é possível prover, quantificar e organizar de forma mais assertiva o estoque.

Portanto, é imprescindível a adequada quantificação de quais materiais e medicamentos são mais usados e consumidos, avaliando a relação desse consumo com a sazonalidade ao qual estão sujeitos, devido a periodicidade de algumas doenças conforme os meses do ano. Esse dimensionamento deve prever os aumentos e diminuições conforme o uso e entrega desses produtos, evitando vencimentos em prateleiras ou falta de medicamentos.

\section{RESULTADO E DISCUSSÃO}

Foram identificadas no presente estudo algumas das principais dificuldades e fatores que causam interferências na gestão dos suprimentos nas farmácias hospitalares, dentre eles estão a falta de planejamento dos medicamento e materiais, gestão participativa não atuante, ausência de metas preestabelecidas e falta de capacitação profissional, como também outras variantes que serão discutidas na sequência.

Os dados de Silva et al. (2013), apontam que nas farmácias pesquisadas, em maior parte delas, não se realizava o planejamento com objetivos e metas, constou-se ainda que faltavam manuais de normas e procedimentos para direcionar as rotinas. Para ele, gerir uma farmácia hospitalar traz em si o dever de propiciar um suporte adequado, com finalidade de obter o primor na oferta da assistência e assegurar a contribuição técnica, científica, clínica e administrativa. 
Conforme Silva et al. (2013), nenhum hospital dentre os estudados inseria os processos e ou etapas de gestão participativa, focada na qualidade e nos resultados, o que difere do modelo de gestão que é recomendado para esse seguimento hospitalar. O modo de gerência participativa quando ajustada à filosofia institucional tem sido apresentado como uma solução efetiva na melhoria do cuidado, mas infelizmente não é o cenário encontrado na maioria dos hospitais pesquisados. Este resultado mostra uma frágil articulação do trabalho da farmácia hospitalar com os outros setores do hospital.

Por outro panorama Infante e Santos (2007), apontam que a falta de regularidade no abastecimento, por vezes a escassez do produto e até a falta do material, são situações que ocorrem frequentemente em serviços públicos de saúde e trazem impactos que atingem negativamente a sua atuação e imagem perante os profissionais e a comunidade.

Esses dados corroboram com os estudo de Andreoli e Dias (2015), que mostram que os impactos negativos ocasionados a partir dos períodos de desabastecimento, causados por disfunções no cumprimento orçamentário não são pequenos, sendo significativos na instituição. Contudo, neste estudo também foram evidenciados os desperdícios e a má utilização de medicamentos, insumos e equipamentos hospitalares, escasso aperfeiçoamento profissional dos servidores da área de abastecimento e a reduzida vigilância quanto ao planejamento logístico nas organizações de saúde.

Falhas na logística podem ocasionar perdas nos recursos tanto humanos como financeiros, perdendo assim eficácia no fornecimento adequado dos medicamentos. A grande quantidade de produtos sem padronização em detrimento dos padronizados desorganiza o serviço de assistência farmacêutica dentro hospital e produz o aumento do consumo de medicamentos que não estão incluso no elenco de produtos, isso ocasiona um possível aumento de perdas de medicamento padrão por ocasião de vencimento do mesmo, e interfere negativamente no planejamento de compras desses medicamento (BARBOSA, 2015). 
Nos estudos de Gonçalves, Novaes e Simonetti (2006), fatores semelhantes a esses são encontrados, quando eles referem que os índices de gastos financeiros com medicamentos, demonstram que os custos crescentes dos setores da saúde se apresentam insustentáveis, tanto as instituições públicas como às organizações privadas de saúde. Planejar e controlar esses gastos são meios que podem assegurar a sobrevivência das instituições hospitalares.

Conforme Pereira (2006), a gestão da cadeia pode oferecer muitas situações onde é possível se obter aumento da produtividade nos estabelecimentos, como consequência disso pode-se reduzir de forma significativa os custos institucionais, e deste modo adicionar maior valoração aos produtos e serviços prestados.

Por outra perspectiva Noronha e Borges (2005), não identificaram por parte dos gerentes ou responsáveis pelo setor de compras, consulta sobre a análise dos aspectos técnicos e/ou epidemiológicos e variações no consumo, para a definição de cortes ou adições no pedido de compra, nem mesmo encontrou preocupação com a qualidade e a quantidade dos produtos, revelando assim que os mesmo não faziam estas avaliações nos hospitais que pesquisaram.

O profissional farmacêutico vincula-se a todas as fases da cadeia logística do medicamento, primordialmente ele atua na fase de aquisição, devendo estar capacitado para realizar a condução das atividades técnicas como padronização, especificação e parecer técnico (SFORSIN et al., 2012).

O não uso de recursos tecnológicos, como programas para controle de estoque, dificulta o processo de gestão dos suprimentos hospitalares, esse ponto foi uma das ineficiências encontradas e um dos pontos frágeis no processo logístico. A administração dos recursos materiais, humanos e financeiros pode ser feita de forma mais precisa e ágil com a utilização da tecnologia da informação (SFORSIN et al., 2012).

Em síntese, compreende-se que para o alcance da qualidade na gestão dos estoques, é necessária uma boa estrutura organizacional, que viabilize as ações do serviço as 
quais demandam procedimentos bem definidos, conhecimentos técnicos/científicos na área, atividades integradas e busca permanente de melhorias nos processos e resultados na gestão logística da farmácia hospitalar.

\section{CONSIDERAÇÕES FINAIS}

O serviço de farmácia hospitalar é responsável por inúmeros processos que causam grande impacto na assistência em saúde. As farmácias hospitalares precisam desempenhar uma série de atividades complexas de modo organizado e eficaz para atender as necessidades dos pacientes sob seus cuidados.

Nessa diversidade de tarefas encontra-se a gestão dos suprimentos, desde sua aquisição até sua disponibilização em estoque local e no seu destino final, sem a devida eficiência e controle desse processo têm-se que os pacientes (consumidores finais dos produtos) estarão sub atendidos, e correndo riscos de saúde, visto que a falha desse processo pode ocasionar um desabastecimento e um determinado paciente pode ficar sem o medicamento e tratamento adequado.

Este estudo, verificou que em grande parte dos hospitais apresentados se tem dificuldade para manter um adequado controle de estoque, isso ocorre por diversos motivos, desde falta de preparo específico aos profissionais que atuam nessa área de gestão dos suprimentos, como dificuldades para quantificar o real consumo, especificidade do material estocado e até falta de recursos financeiros em algumas situações.

A partir disso, considerou-se a atividade logística de gestão da cadeia de abastecimento como indispensável ao bom funcionamento da farmácia hospitalar, de forma a promover um adequado controle do estoque, abastecimento satisfatório e, portanto, mais eficácia e segurança a todo hospital e ao paciente.

Recomenda-se que mais estudos sejam feitos nessa área e que estes possam contribuir para o primor da assistência prestada pelo serviço de farmácia hospitalar, de modo a trazer benefícios aos hospitais, usuários e sociedade 


\section{REFERÊNCIAS}

ACCIOLY, Felipe; AYRES Antônio de Pádua Salmeron; SUCUPIRA, Cezar. Gestão de estoques. $1^{\circ}$ Ed. Rio de Janeiro: Editora FGV, 2008, pg 40-47, volume 1.

ANDREOLI, Gustavo Luís Meffe; DIAS Cleidson Nogueira. Planejamento e Gestão Logística de Medicamentos em uma Central de Abastecimento Farmacêutico Hospitalar. Revista de Administração Hospitalar e Inovação em Saúde - RAHIS v.12 n.4(2015).

BARBIERI, José Carlos.; MACHLINE, Claude. Logística hospitalar: teoria e prática. 3o Ed. São Paulo: Saraiva, 2017.

BARBOSA, Katia Simoni da Silva. Gerenciamento de Farmácia Hospitalar: Otimização da Qualidade, Produtividade E Recursos Financeiros. Revista Saúde e Desenvolvimento vol. 7, n.4 jan - dez 2015. Disponível: https://www.uninter em:.com/revistasaude/index.php/saudeDesenvolvimento/article/view/343. Acesso em: 12 jul. 2019.

BARBUSCIA, Caloger Sauveur (2006). Gestão de suprimentos na administração hospitalar pública. In: GONÇALVES, Ernesto Lima. Gestão Hospitalar: Administrando o hospital moderno. (Cap. 9, pag. 196-224). São Paulo: Saraiva, 2012.

CARVALHO, José Crespo De; RAMOS, Tânia. Logística na Saúde. $3^{\mathrm{a}}$ Ed. Lisboa: Edições Sílabo, 2009.

CAVALLINI, Mírian Elias; BISSON, Marcelo Polacow. Farmácia hospitalar. $2^{\circ}$ Ed. Barueri: Manole, 2010.

Conselho Federal de Farmácia. Resolução no 492, de 26 de novembro de 2008. Regulamenta o exercício profissional nos serviços de atendimento pré-hospitalar, na farmácia hospitalar e em outros serviços de saúde, de natureza pública ou privada. Brasília: CFF, 2008. Disponível em: https://cff-br.implanta.net.br/ 
PortalTransparencia/Publico/ArquivosAnexos/Download?idArquivoAnexo=774c5fd93efd-411e-8eed-ec4206533b1c Acesso em:13 jul. 2019.

FARIAS, Diego Carlos; ARAUJO, Fernando Oliveira de. Gestão hospitalar no Brasil: revisão da literatura visando ao aprimoramento das práticas administrativas em hospitais. Ciênc. saúde coletiva, Rio de Janeiro, v. 22, n6, p.189a1904, jun.2017. Disponível em: http://www.scielo.br/scielo.php? scriptsci _arttext\&pid=S141381232017002601895\&lng=en\&nrm=iso. Acesso em: 23 ago. 2019.

FERRANTI, Eliane. Gestão de estoque de medicamentos utilizando classificação ABC em um hospital público. Perspectiva Econômica, vol. 13, n. 3, p. 215-229, n. especial: dez. 2017.

GONÇALVES Antônio Augusto; NOVAES, Mario Lúcio de Oliveira; SIMONETTI, Vera Maria Medina. Otimização de farmácias hospitalares: eficácia da utilização de indicadores para gestão de estoques. In: Encontro Nacional de Engenharia de Produção, 26., 2006, Fortaleza-CE. Anais eletrônicos... Rio de Janeiro: ABPRO, 2006. p. 2- 8. Disponível em: http://www.abepro.org.br/biblioteca/ENEGEP2006 _TR450302_7149.pdf. Acessado em: 4 ago. 2019.

INFANTE, Maria; SANTOS, Maria Angélica Borges dos. A organização do abastecimento do hospital público a partir da cadeia produtiva: uma abordagem logística para a área de saúde. Ciência \& Saúde coletiva [online]. 2007, vol.12, n.4, pp. 945-954. ISSN 1413-8123. Disponível em: <https://www.scielo.br/ scielo.php?pid=S1413-81232007000400016\&script=sci_abstract\&tlng=pt> .Acesso em: 30 ago. 2019.

NETO, Gonzalo Vecina; FILHO, Wilson Reinhardt. Gestão de Recursos Materiais e de Medicamentos. $1^{\circ}$ Ed. São Paulo: Faculdade de Saúde Pública da Universidade de São Paulo, 1998. V 12 (Série Saúde \& Cidadania). Disponível em: $<$ http://colecoes.sibi.usp.br/fsp/items/show/2372\#? c=0\&m=0\&s=0\&cv=0>. Acesso em 3 ago. 2019. 
NORONHA, André Gustavo Gadelha Mavignier de; BORGES, Djalma Freire. Qualidade da gestão de medicamentos em hospitais públicos. RAP, v. 39, n. 4, 895-912, jul./ago. 2005.

NOVAES, Marcio Lucio de Oliveira; SIMONETTI, Vera Maria Medina; AFONSO, Marina Weil. Gestão de suprimentos da farmácia hospitalar com a implantação de métodos gerenciais de insumos usados em manufatura. Revista eletrônica Produção \& Engenharia. V.2 n. 1p.57-68, jan/jul. 2009. Disponível em: https://periodicos.ufjf.br/index.php/producaoeengenharia/article/view/28945. Acesso em: Acesso em 3 ago. 2019.

OLIVEIRA, Thomás Silva; MUSETTI, Marcel Andreoli. Revisão compreensiva de logística hospitalar: conceitos e atividades. Revista de Gestão em Sistemas de Saúde - RGSS Vol. 3, N. 1. janeiro/junho. 2014.

PEREIRA, Moacir. Um modelo de gestão de abastecimento de medicamentos em farmácia hospitalar. 2006.Tese (Doutorado em Engenharia de Produção) Faculdade de Engenharia Arquitetura e Urbanismo - UNIMEP, Santa Bárbara D’Oeste, 2006).

PLATT, Allan Augusto. Logística e cadeia de suprimentos. $3^{\circ}$ ed. - Florianópolis: Departamento de Ciências da Administração/UFSC, 2015. Disponível em: http://arquivos.eadadm.ufsc.br/ EaDADM/UAB320132/ Modulo_6/Logistica/_ material_didatico/logistica_e_cadeia_de_suprimentos-3ed-online.pdf. Acesso em: 12 jul. 2019.

PRESTES, Andréa. Manual do gestor hospitalar. Organizadores: Andréa Prestes, José Antônio Ferreira Cirino, Rosana Oliveira e Viviã de Sousa.1ํEd. - Brasília: Federação Brasileira de Hospitais, 2019.

REIS Adriano Max Moreira; PERINI Edson. Desabastecimento de medicamentos: determinantes, consequências e gerenciamento. Ciências e Saúde Coletiva. 2008, vol. 13, p. $603-610$. 
SILVA, Mario Jorge Sobreiro da; MAGARINOS-TORRES, Rachel; OLIVEIRA, Maria Auxiliadora; OSORIO-de-CASTRO, Cláudia Garcia Serpa. Avaliação dos serviços de farmácia dos hospitais estaduais do Rio de Janeiro, Brasil. Ciência \& Saúde Coletiva, n18(12): p. 3605 - 3620, 2013.

SILVA, Valdete Lourenço. Defict no Processo de Logística Hospitalar Desafio para uma Gestão Eficiente no Contexto da Saúde Pública Brasileira. Revista Científica Multidisciplinar Núcleo do Conhecimento. Ano 03, Ed. 08, Vol. 16, pp. 232-244, agosto de 2018. ISSN:2448-0959. Disponível em: https://www.nucleodoconhecimento.com.br/saude/logistica. Acesso em: 12 jul 2019.

SOUSA, Andréa Modesto. Logística Hospitalar: a eficiência do processo de suprimento de medicamentos na rede pública hospitalar do Distrito Federal. 2011. p. 39. Monografia apresentada a Universidade de Brasília-UNB, para obtenção do grau de bacharel em Administração.

SFORSIN, Andréa Cassia Pereira et al. Gestão de compras em farmácia hospitalar. Revista Farmácia Hospitalar, n. 16, mar/abr/mai. 2012. Disponível em: https://www.cff.org.br/sistemas/geral/revista/pdf/137/encarte_farmAcia_hospitalar_85 .pdfAcesso em: 9 jul. 2019.

Enviado: Agosto, 2020.

Aprovado: Setembro, 2020. 